\title{
SHORT TIMESCALE VARIATIONS OF THE H $\alpha$ DOUBLE-PEAKED PROFILE OF THE NUCLEUS OF NGC 1097
}

\author{
Jaderson S. Schimoia ${ }^{1}$, Thaisa Storchi-Bergmann ${ }^{1}$, Rodrigo S. Nemmen ${ }^{2}$, Cláudia Winge $^{3}$, and Michael Eracleous ${ }^{4}$ \\ ${ }^{1}$ Instituto de Física, Universidade Federal do Rio Grande do Sul, Campus do Vale, Porto Alegre, RS, Brazil; silva.schimoia@ufrgs.br \\ ${ }^{2}$ NASA Goddard Space Flight Center, 8800 Greenbelt Road, Greenbelt, Maryland, USA \\ ${ }^{3}$ Gemini South Observatory, c/o AURA Inc., Casilla 603, La Serena, Chile \\ ${ }^{4}$ Department of Astronomy and Astrophysics, the Pennsylvania State University, 525 Davey Lab, University Park, PA 16802, USA \\ Received 2011 October 6; accepted 2012 January 24; published 2012 March 16
}

\begin{abstract}
The broad (FWHM $\sim 10,000 \mathrm{~km} \mathrm{~s}^{-1}$ ) double-peaked H $\alpha$ profile from the LINER/Seyfert 1 nucleus of NGC 1097 was discovered in 1991 and monitored for the following 11 years. The profile showed variations attributed to the rotation of gas in a non-axisymmetric Keplerian accretion disk, ionized by a varying radiatively inefficient accretion flow (RIAF) located in the inner parts of the disk. We present and model 11 new spectroscopic observations of the double-peaked profile taken between 2010 March and 2011 March. This series of observations was motivated by the finding that in 2010 March the flux in the double-peaked line was again strong, indeed, in 2010 December, even stronger than in the observations of a decade ago. We also discovered shorter timescale variations than in the previous observations: (1) the first, of $\sim 7$ days, is interpreted as due to "reverberation" of the variation of the ionizing source luminosity, and the timescale of 7 days as the light crossing time between the source and the accretion disk; this new timescale and its interpretation provides a distance between the emitting gas and the supermassive black hole and as such introduces a new constraint on its mass; (2) the second, of $\approx 5$ months, was attributed to the rotation of a spiral arm in the disk, which was found to occur on the dynamical timescale. We use two accretion disk models to fit theoretical profiles to the new data, both having non-axisymmetric emissivities produced by the presence of an one-armed spiral. Our modeling constrains the rotation period for the spiral to be $\approx 18$ months. This work supports our previous conclusion that the broad double-peaked Balmer emission lines in NGC 1097-and probably also in other low-luminosity active nuclei-originate from an accretion disk ionized by a central RIAF.
\end{abstract}

Key words: accretion, accretion disks - galaxies: individual (NGC 1097) - galaxies: nuclei - galaxies: Seyfert line: profiles

Online-only material: color figures

\section{INTRODUCTION}

The barred spiral galaxy NGC 1097 has an active nucleus classified as LINER (Low-Ionization Nuclear Emission-Line Region; Heckman 1980), whose spectrum was found in 1991 to show transient broad $\left(\mathrm{FWHM} \approx 10,000 \mathrm{~km} \mathrm{~s}^{-1}\right.$ ) double-peaked Balmer lines (Storchi-Bergmann et al. 1993). The nucleus of NGC 1097 was the first low-luminosity active galactic nucleus (LLAGN) in a LINER discovered displaying such lines, and, because of these broad lines, has also been classified as a Seyfert 1 nucleus. Later, other LLAGNs in LINER nuclei were found to display double-peaked Balmer lines, such as M 81 (Bower et al. 1996), NGC 4203 (Shields et al. 2000), and NGC 4450 (Ho et al. 2000).

The double-peaked $\mathrm{H} \alpha$ profile of the NGC 1097 nucleus was monitored for the following 11 years after the discovery (Storchi-Bergmann et al. 2003; SB03), showing variability in its flux, width, and relative intensity of the blue and red peaks. These variations were attributed to gas emission from a thin Keplerian accretion disk, with a non-axisymmetric perturbation around a supermassive black hole (hereafter SMBH), where the gas is ionized by a central source, (Eracleous et al. 1995; Storchi-Bergmann et al. 1995, 1997, 2003).

The variability of the double-peaked profile of NGC 1097 is not rare. The first active nucleus found to display variable double-peaked broad Balmer emission lines was the nucleus of the radio galaxy 3C 390.3 (Yee \& Oke 1981). This object can be considered prototypical of the class of double-peaked emitters, because many of the now well known features of these objects, such as variability in the relative intensity of the blue and red peaks (Veilleux et al. 1991; Zheng et al. 1991), were first observed in 3C 390.3. Another remarkably well studied double-peaked emitter is the active nucleus of Arp 102B. For this object, a study by Chen \& Halpern (1989) has shown that the double-peaked Balmer lines cannot be driven by local viscous dissipation in the line-emitting part of the disk, as the $\mathrm{H} \alpha$ luminosity is of the order or even exceeds the energy locally available to power the line. The same conclusion was obtained for other double-peaked emitters by Eracleous \& Halpern (1994) and Strateva et al. $(2006,2008)$. These authors suggested that the lines are powered by illumination from an external source. The ionizing source could be an geometrically thick accretion flow, located within the inner radius of the line-emitting portion of the disk. In the case of NGC 1097, Nemmen et al. (2006) found that the spectral energy distribution (SED) of the nucleus is indeed well described by such a structure, a radiatively inefficient accretion flow (RIAF; Narayan \& McClintock. 2008). This has led to the conclusion that an inner RIAF is the ionizing source of the accretion disk in NGC 1097.

Lewis et al. (2010) and Gezari et al. (2007) reported a longterm monitoring of 14 other double-peaked emitters and found similar variations to those observed for NGC 1097, 3C390.3 and Arp 102B. These objects also display in at least one epoch the red peak more intense than the blue peak. This indicates that most line-emitting disks are non-axisymmetric, as in an axisymmetric disk the blue peak should be always more intense than the red because of Doppler boosting. The disks could then be elliptical (Eracleous et al. 1995), or have a non-axisymmetric 
emitting structure, such as a hot spot or spiral arm (Lewis et al. 2010).

Another model that can reproduce double-peaked emissionline profiles is the one proposed by Gaskell et al. (2007) and Gaskell (2010) in which the ionizing source is the continuum from a thin accretion disk, and the origin of the double-peaked lines is the broad-line region (BLR) that is proposed to be an inner extension of the torus, in the shape of a "bird's nest." This geometry is not that distinct from that of the outer parts of an accretion disk, and in this regard is in approximate agreement with our proposed geometry. But, in the case of NGC 1097, the study by Nemmen et al. (2006) favors an RIAF origin for the nuclear ionizing continuum instead of a hot and thin accretion disk.

The monitoring of double-peaked profiles is a valuable experiment in the investigation of the structure and dynamics of the accretion disks and how they evolve as the gas moves inward to feed the SMBH. In Storchi-Bergmann et al. (2003), observing the NGC $1097 \mathrm{H} \alpha$ double-peaked profile approximately once or twice a year, we concluded that the asymmetry of the doublepeaked profile was due to a spiral arm perturbation in the disk, rotating with a period of 5.5 years. But as relevant timescales for evolution of the innermost regions of AGNs can be as short as weeks and months, we decided to repeat our experiment on NGC 1097 in order to probe shorter timescales. Here we report the results of this experiment: we indeed found short variability timescales. We also verified that, although our last observations of 2001 showed that the profile was becoming fainter and fainter, it is now again strong and was even stronger in 2010 December than in all previous observations.

This paper is organized as follows: in Section 2 we describe the observations and the data reduction, in Section 3 we present the observational results, in Section 4 we describe the models and the results from the modeling. In Section 5 we discuss the timescales of the accretion disk variability and the interpretation of the modeling. The conclusions of this work are presented in Section 6.

\section{OBSERVATIONS AND DATA REDUCTION}

Long-slit optical spectra of the nucleus of NGC 1097 were obtained in queue mode in 11 epochs from 2010 March 4 to 2011 March 21, using the Gemini South Multi-Object Spectrograph (GMOS) in the long-slit mode. The allocation was part of the "Poor Weather" opportunity, which is ideal for spectral monitoring which does not need absolute flux calibration or regular cadence. This is the case for the nucleus of NGC 1097, for which we use the integrated flux in the narrow emission lines to normalize the spectra to match previous observations, under the assumption that the narrow lines do not vary within the extraction aperture.

The first three observations were obtained at time intervals of 4-5 months, since our previous monitoring suggested significant variations on a timescale of years. However, our new observations showed strong variations in the nuclear spectrum on timescales of months, which motivated us to ask for new observations at shorter time intervals. We were then able to get at least two observations a month from 2011 January to 2011 March, as shown in the observing log presented in Table 1. This table lists the identification of the program, date of observation, position angle (p.a.) of the slit, and the average seeing during the observations, measured as the FWHM of a spatial profile extracted along a range of columns containing only the broad line (after subtraction of the underlying continuum).
Table 1

Observation Log

\begin{tabular}{llcc}
\hline \hline Program ID & UT Date & $\begin{array}{c}\text { p.a. } \\
\text { (deg) }\end{array}$ & $\begin{array}{c}\text { Seeing } \\
\text { (arcsec) }\end{array}$ \\
\hline GS-2009B-Q-99 & 2010 Mar 4 & 70 & 1.00 \\
GS-2010A-Q-81 & 2010 Aug 15 & 55 & 0.98 \\
GS-2010B-Q-90 & 2010 Dec 22 & 280 & 0.63 \\
GS-2010B-Q-90 & 2011 Jan 11 & 280 & 0.73 \\
GS-2010B-Q-90 & 2011 Jan 15 & 280 & 0.73 \\
GS-2010B-Q-90 & 2011 Jan 18 & 280 & 0.70 \\
GS-2010B-Q-90 & 2011 Feb 8 & 280 & 1.21 \\
GS-2010B-Q-90 & 2011 Feb 10 & 280 & 0.79 \\
GS-2010B-Q-90 & 2011 Feb 14 & 280 & 0.72 \\
GS-2010B-Q-90 & 2011 Mar 17 & 280 & 1.43 \\
GS-2010B-Q-90 & 2011 Mar 21 & 280 & 0.82 \\
\hline
\end{tabular}

The data were obtained with the GMOS South with a slit width of $1^{\prime \prime}$.0 ( $~ 80 \mathrm{pc}$ ), using the B600 grating and the GG455 filter to block possible higher order contamination. The spectral range covered was $6000-7000 \AA$ for most spectra, at a spectral resolution of $\sim 4.5 \AA$ (FWHM of the lines in the arc spectrum, $\approx 200 \mathrm{~km} \mathrm{~s}^{-1}$ ). Six $600 \mathrm{~s}$ exposures were obtained at each epoch, which were then combined into a final spectrum.

The slit width of 1".0 was selected because it is usually larger than the average seeing during the observations and was also the slit width of previous observations (SB03). Using the same slit width allows us to normalize the new spectra so that they match the flux of the narrow emission lines observed in previous spectra and study the evolution of the flux and profile of the broad $\mathrm{H} \alpha$ line over $\approx 20 \mathrm{yr}$.

The data reduction was performed using the standard tasks from the gmos package in the Image Reduction and Analysis Facility software (IRAF ${ }^{5}$ ). We extracted the nuclear spectra using a window of $1^{\prime \prime} .0 \times 1^{\prime \prime} .0$ centered at the peak of the continuum emission, assumed to correspond to the galaxy nucleus. This position coincides also with the location of the unresolved source of the double-peaked $\mathrm{H} \alpha$ lines. Figure 1 shows the acquisition image of the observation of 2010 August 15, where the nuclear extraction region is represented by the square labeled A.

\subsection{Stellar Population Contribution}

Since the nuclear spectra show strong absorption lines from the underlying stellar population, we subtracted the stellar population contribution, in order to isolate and better study the emission line profile and in particular the broad $\mathrm{H} \alpha$ line. A template stellar population spectrum was obtained as follows. For each epoch, in addition to the nuclear spectrum, we extracted two more spectra using two similar windows of $1^{\prime \prime} .0 \times 1$." 0 , one centered at $1^{\prime \prime} .5$ to the southwest (Figure 1, region B) and another at $1^{\prime \prime} .5$ to the northeast of the nucleus (Figure 1, region $\mathrm{C}$ ). These extranuclear spectra do not show any broad $\mathrm{H} \alpha$ emission, but show some narrow-line emission, which was edited out, using as guides, stellar population templates from previous studies (Bica et al. 1988). Averaging these two spectra, we obtain a stellar population spectrum from a location approximately $100 \mathrm{pc}$ away from the nucleus. Under the assumption that this spectrum is representative of the stellar population at the nucleus, we subtracted it from the nuclear spectrum (after normalizing the

\footnotetext{
5 IRAF is distributed by the National Optical Astronomy Observatory, which is operated by the Association of Universities for Research in Astronomy, Inc., under cooperative agreement with the National Science Foundation.
} 


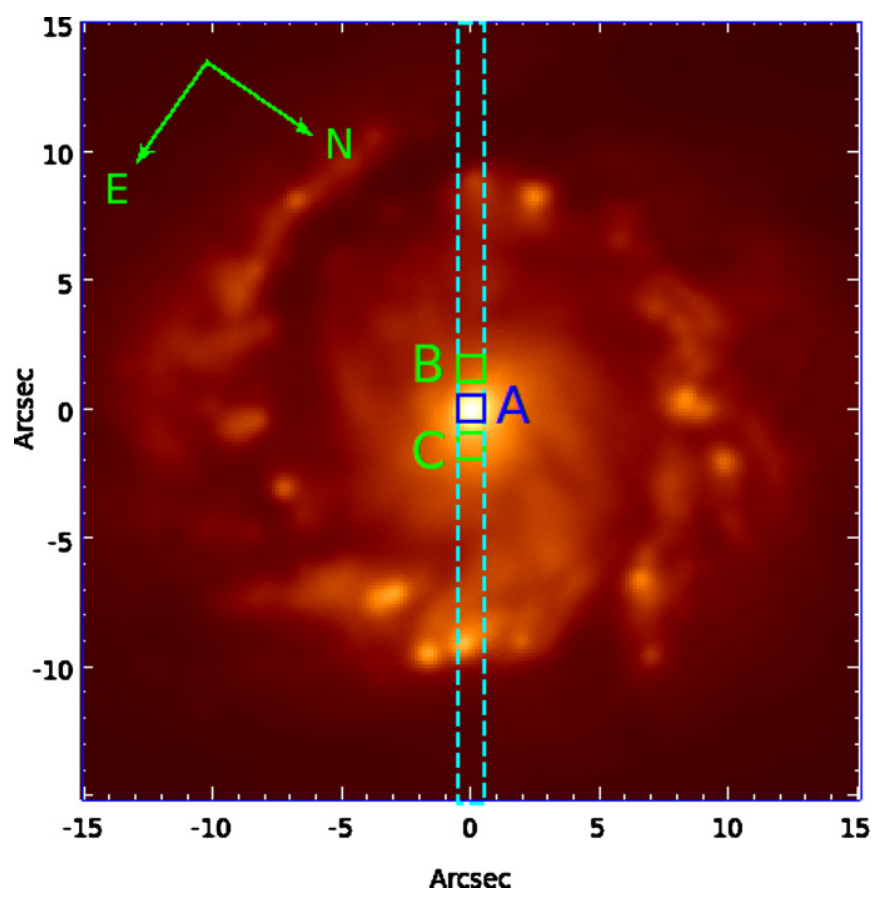

Figure 1. Acquisition image from 2010 August 15, where we illustrate the locations of the $1^{\prime \prime} .0 \times 1^{\prime \prime} .0$ extraction windows for the nucleus and stellar population spectra. The square labeled A represents the nuclear extraction window, while squares $B$ and $C$ represent the extranuclear ones, from which we obtained the spectrum of the underlying stellar population.

(A color version of this figure is available in the online journal.)

continuum to the corresponding value at the nucleus) thus isolating the gas emission.

Figure 2 illustrates the process for the spectrum of 2010 August 15, which covers a broader spectral range than most spectra, from 4750 to $7200 \AA$. We note that this process reveals the presence of a broad double-peaked $\mathrm{H} \beta$ line that was not obvious prior to the subtraction, as well as some weak emission lines such as [NI] $\lambda 5199 \AA$ and [O I] $\lambda 6363 \AA$. The Na I D absorption line in the residual spectrum is most probably interstellar, as all other stellar absorption lines did not show any residual.

We also note that after subtracting the stellar population spectrum from the nuclear one, we do not see any evidence for a significant contribution of an AGN continuum, as can be seen in Figure 2. The effect of a nuclear continuum, if present, would be to dilute the stellar absorption lines, making them shallower at the nucleus than outside. The subtraction of the stellar population template, as described above, after normalization to the flux in the continuum of the nuclear spectrum would result in the presence of "fake" emission features in the residual spectrum and a possible slope (in the case of a blue continuum, for example), which are not seen.

We have performed a sequence of tests in order to estimate the contribution from a featureless continuum (FC) that could be "hidden" in the nuclear spectrum as follows. We added the contribution of the FC, assumed in the form $F_{v} \propto v^{1.5}$, to the stellar population template described above, at increasing percent contributions of its flux at $5800 \AA$. The resulting composite (FC+stellar population) spectrum was then renormalized to the same flux at $5800 \AA$, and we subtracted the stellar population template from it. We concluded that for the short wavelength range and the signal-to-noise ratio of our observations, in order to have clear residuals showing fake emission features and a

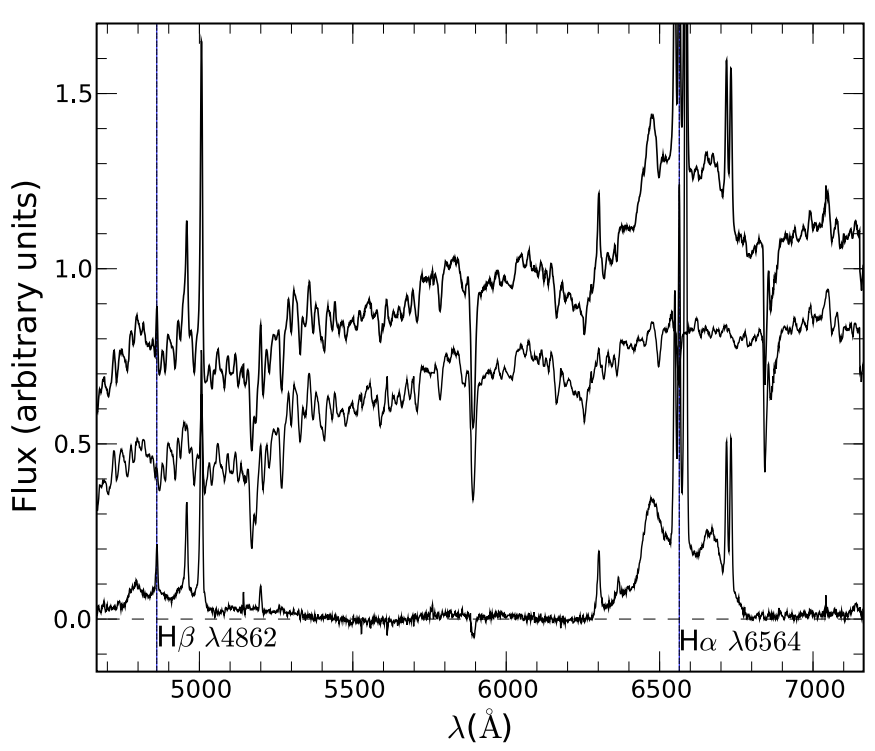

Figure 2. Observation from 2010 August 15. Top: nuclear spectrum (region A of Figure 1). Middle: stellar population obtained from averaging the spectra from regions $\mathrm{B}$ and $\mathrm{C}$ in Figure 1. Bottom: nuclear spectrum after the subtraction of the stellar population.

blue continuum we need the FC continuum to contribute with at least $15 \%-20 \%$ of the flux at $5800 \AA$. We thus can say that a possible FC contribution to the nuclear spectrum is lower than $20 \%$ of the continuum flux at $5800 \AA$. We consider this value an upper limit, as a comparison between the stellar continuum from our spectra and the AGN continuum obtained in the work of Nemmen et al. (2006) shows that the stellar continuum in our aperture is about 30 times stronger than the AGN continuum in the optical.

\section{RESULTS}

After subtracting the contribution of the stellar population, the final step before the analysis of the double-peaked profile was to normalize the spectra using as reference a previous flux-calibrated spectrum. We used as reference the spectrum of 1991 November 2 (Storchi-Bergmann et al. 1993), and assumed that the flux of the narrow lines $\mathrm{H} \alpha$, [N II] $\lambda \lambda 6548,6584$, and [S II] $\lambda \lambda 6717,6731$ did not vary during the last 20 years. This is a common assumption in this type of study, as the narrow lines originate in extended regions with spatial scales much larger $(\sim 100 \mathrm{pc})$ than that of the accretion disk $\left(\sim 10^{-3} \mathrm{pc}\right)$. Thus, their response time to variations in the ionizing flux is correspondingly much larger than that for the line-emitting accretion disk, and cannot respond to variations of the ionizing flux that are as fast as those we have detected.

The normalized spectra from our 2010-11 observations are compared with the reference spectrum of 1991 November in Figure 3 (pairs of spectra are shifted from each other in the vertical direction for clarity). In this figure we show the three spectra from 2011 January, the three from 2011 February, and the two from 2011 March, respectively, on top of each other because we did not find any significant variation within each group.

Note that the double-peaked $\mathrm{H} \alpha$ profile from 2010 August is very different from that of 2010 March. In 2010 March, the red peak was stronger than the blue peak, while in 2010 August, the blue peak was stronger than the red. This inversion of the relative intensity of the peaks occurred on a timescale of 


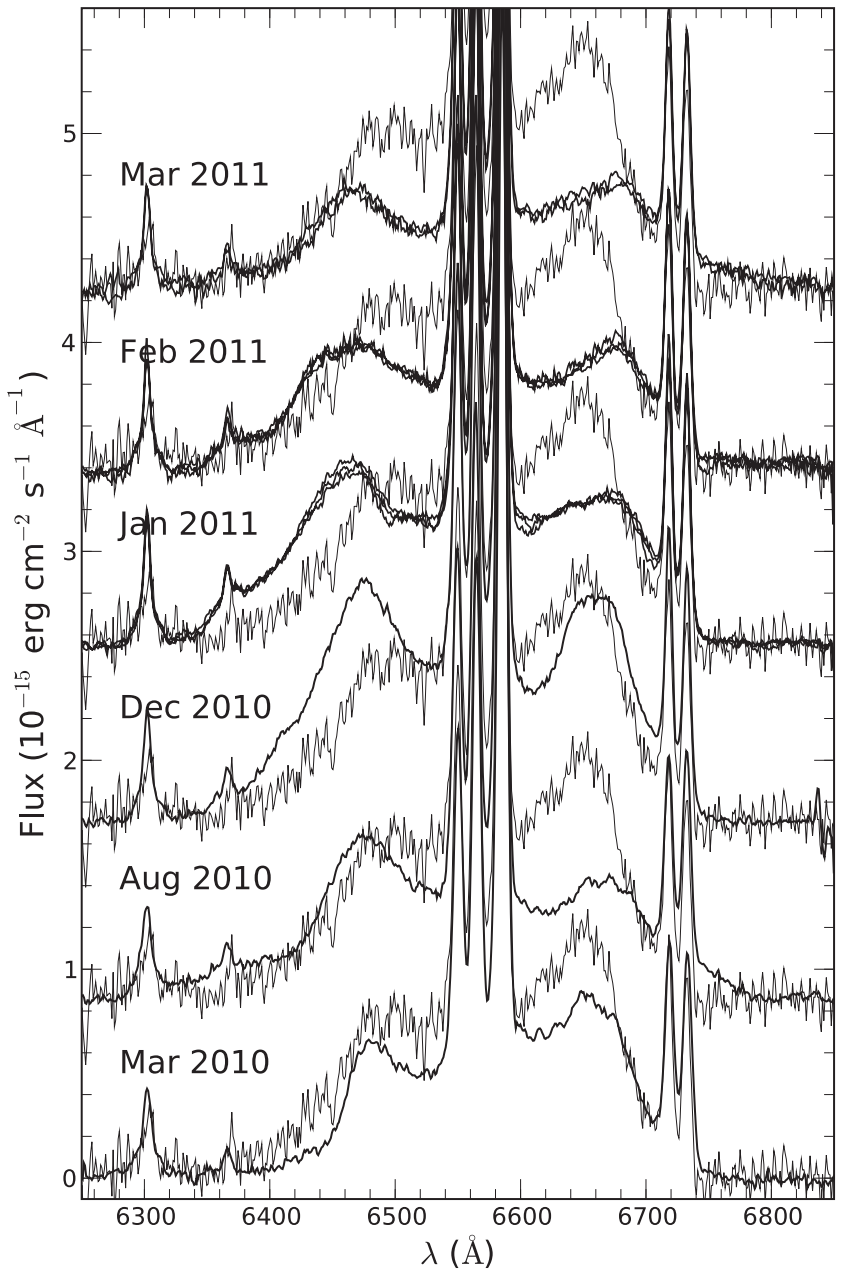

Figure 3. Nuclear spectra from 2010 March to 2011 March each compared with the reference spectrum of 1991 November 2 (gray). For 2011 January-March we show the spectra obtained a few days apart plotted on top of each other, as no significant changes were found between them.

5 months. In addition, in 2010 December, the profile showed a marked increase in its integrated flux compared with that of 2010 August, while the red and blue peaks showed similar strengths.

From 2010 December 22 to 2011 January 11 we observed a $\sim 25 \%$ decrease in the integrated flux of the double-peaked profile. The three observations of 2011 January were obtained within a time interval of seven days, and showed no significant variations.

In 2011 February, the three observations were obtained within a time interval of six days, and again we did not see significant variations among the three profiles. However, the total flux continued to decrease compared with that of January, with the blue and red peaks showing similar strengths.

In 2011 March, we obtained two observations separated by a time interval of four days, between which we again did not find significant variation in the double-peaked profile. But the total flux decreased further, and the profile showed the red peak somewhat stronger than the blue peak.

In summary, this series of observations obtained between 2010 March and 2011 March reveal: (1) a marked increase of the broad-line flux between 2010 August and 2010 December; (2) a significant decrease of the flux on timescales longer than a week; (3) no significant changes in the profile on time intervals shorter than a week; (4) an inversion in the relative strength of the blue and red peaks on a timescale of 5 months.

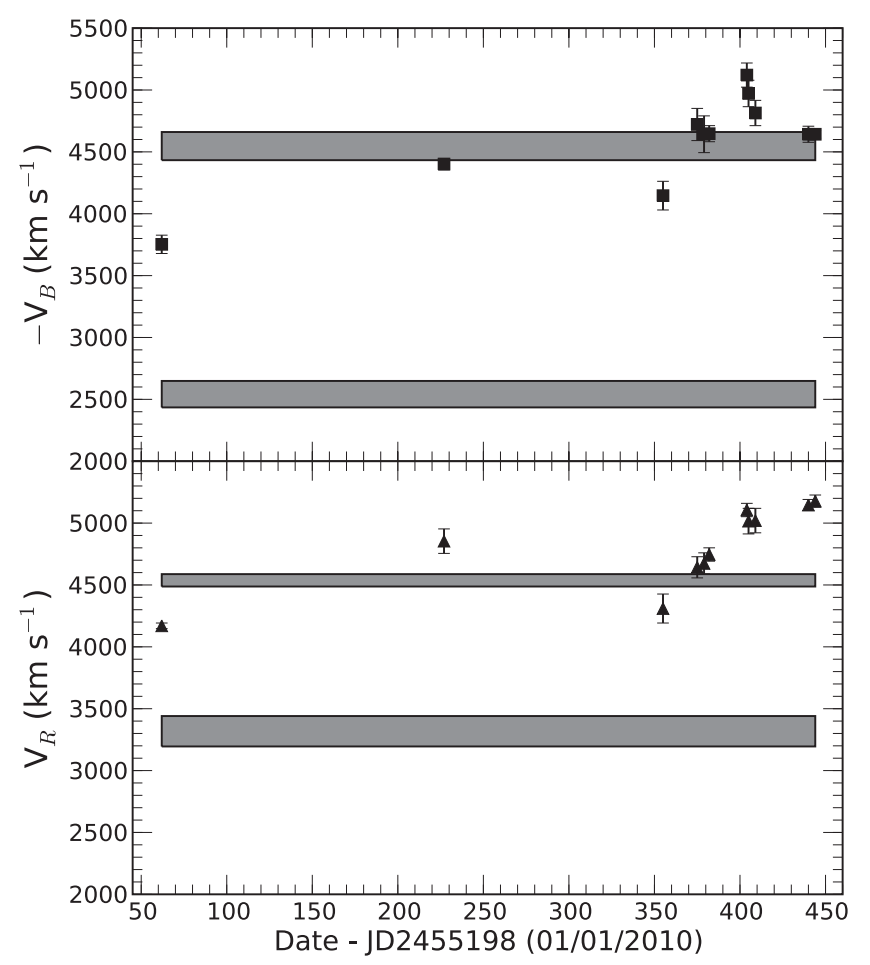

Figure 4. Evolution of the peak velocities of the blue (top panel) and red (bottom panel) peaks from 2010 March to 2011 March. The gray horizontal bars show the minimum and maximum values (plus uncertainties) in the spectra from 1991 to 2001 (SB03).

\subsection{Measurements of the Double-peaked Profile Characteristics}

We have quantified the observed variations by measuring the wavelengths of the blue and red peaks of the profiles, their peak flux densities, and the integrated flux of the broad line, as described below. We list the results in Table 2 .

In order to isolate the broad double-peaked $\mathrm{H} \alpha$ emission and perform the measurements, we first evaluated the contribution of the narrow lines $\mathrm{H} \alpha_{\text {narrow }} \lambda 6564$, [N II] $\lambda \lambda 6549,6584$, [S II] $\lambda \lambda 6718,6732$ and [O I] $\lambda \lambda 6302,6365$ by fitting Gaussians to these lines and then subtracting them. The flux of the doublepeaked line $\left(F_{\text {broad }}\right)$ was measured by integrating the total flux under the profile after this subtraction. The average uncertainty in $F_{\text {broad }}$ is $1 \times 10^{-14} \mathrm{erg} \mathrm{cm}^{-2} \mathrm{~s}^{-1}$.

The wavelengths of the blue and red peaks $\left(\lambda_{B}\right.$ and $\left.\lambda_{R}\right)$ and the corresponding peak flux densities $\left(F_{B}\right.$ and $\left.F_{R}\right)$ were obtained by fitting three Gaussians to the double-peaked profile, one for the blue peak, another for the red peak and a third Gaussian for the center of the profile. $F_{B}$ and $F_{R}$ are the peak flux densities of the Gaussians fitted to the blue and red peaks, respectively, while $\lambda_{B}$ and $\lambda_{R}$ are the corresponding wavelengths. We have tried other methods as well, such as using splines to fit the peaks, but concluded that the fit of Gaussians gave equivalent but more robust results. The average uncertainties in the measurements are $\approx 4 \AA$ for $\lambda_{B}$ and $\lambda_{R}$ and $\approx 6 \times 10^{-17} \mathrm{erg} \mathrm{cm}^{-2} \mathrm{~s}^{-1} \AA^{-1}$ for $F_{B}$ and $F_{R}$.

From $\lambda_{B}$ and $\lambda_{R}$ we obtained the blue and red peak velocities $V_{B}$ and $V_{R}$ relative to the systemic velocity of the galaxy, adopted as the one corresponding to the narrow component of the $\mathrm{H} \alpha$ emission, as we have done in our previous studies (SB03). Figure 4 shows the temporal evolution of $V_{B}$ and $V_{R}$. The velocities of the blue peak range between -5500 and $-3500 \mathrm{~km} \mathrm{~s}^{-1}$, while for the red peak they are between 4000 and 
Table 2

Measurements of the Profile Properties

\begin{tabular}{|c|c|c|c|c|c|}
\hline Date & $\begin{array}{l}\lambda_{B}{ }^{\mathrm{a}} \\
(\AA)\end{array}$ & $\begin{array}{l}\lambda_{R}{ }^{\mathrm{a}} \\
(\AA)\end{array}$ & $\begin{array}{c}F_{B}^{\mathrm{b}} \\
\left(10^{-15} \mathrm{erg} \mathrm{cm}^{-2} \mathrm{~s}^{-1} \AA^{-1}\right)\end{array}$ & $\begin{array}{c}F_{R}^{\mathrm{b}} \\
\left(10^{-15} \mathrm{erg} \mathrm{cm}^{-2} \mathrm{~s}^{-1} \AA^{-1}\right)\end{array}$ & $\begin{array}{c}F_{\text {broad }^{\mathrm{c}}} \\
\left(10^{-15} \mathrm{erg} \mathrm{cm}^{-2} \mathrm{~s}^{-1}\right)\end{array}$ \\
\hline 2010 Mar 4 & $6483.0 \pm 1.6$ & $6656.5 \pm 0.5$ & $0.760 \pm 0.06$ & $0.970 \pm 0.02$ & $197.4 \pm 7.7$ \\
\hline 2010 Aug 15 & $6469.0 \pm 1.0$ & $6671.7 \pm 2.2$ & $0.760 \pm 0.06$ & $0.570 \pm 0.03$ & $186.1 \pm 4.4$ \\
\hline 2010 Dec 22 & $6474.5 \pm 2.5$ & $6659.6 \pm 2.6$ & $1.160 \pm 0.02$ & $1.070 \pm 0.02$ & $258.5 \pm 7.2$ \\
\hline 2011 Jan 11 & $6462.1 \pm 2.8$ & $6667.0 \pm 1.9$ & $0.830 \pm 0.01$ & $0.700 \pm 0.02$ & $210.1 \pm 6.2$ \\
\hline 2011 Jan 15 & $6463.8 \pm 3.2$ & $6667.7 \pm 1.9$ & $0.840 \pm 0.01$ & $0.710 \pm 0.01$ & $216.7 \pm 4.0$ \\
\hline 2011 Feb 8 & $6453.5 \pm 2.1$ & $6677.3 \pm 1.2$ & $0.550 \pm 0.03$ & $0.580 \pm 0.02$ & $153.2 \pm 1.5$ \\
\hline $2011 \mathrm{Feb} 10$ & $6456.7 \pm 2.3$ & $6675.3 \pm 2.3$ & $0.600 \pm 0.05$ & $0.600 \pm 0.03$ & $162.7 \pm 1.8$ \\
\hline 2011 Feb 14 & $6460.1 \pm 2.2$ & $6675.4 \pm 2.2$ & $0.570 \pm 0.01$ & $0.580 \pm 0.02$ & $150.0 \pm 5.2$ \\
\hline 2011 Mar 17 & $6463.8 \pm 1.4$ & $6678.2 \pm 1.0$ & $0.490 \pm 0.01$ & $0.520 \pm 0.03$ & $129.2 \pm 3.0$ \\
\hline 2011 Mar 21 & $6463.8 \pm 0.7$ & $6678.9 \pm 1.1$ & $0.470 \pm 0.01$ & $0.500 \pm 0.02$ & $127.1 \pm 3.2$ \\
\hline
\end{tabular}

Notes.

${ }^{a} \lambda_{B}$ and $\lambda_{R}$ are the peak wavelength of the blue and red peaks, respectively.

${ }^{\mathrm{b}} F_{B}$ and $F_{R}$ are the corresponding peak fluxes.

${ }^{\mathrm{c}} F_{\text {broad }}$ is the integrated flux of the broad double-peaked $\mathrm{H} \alpha$ emission line.

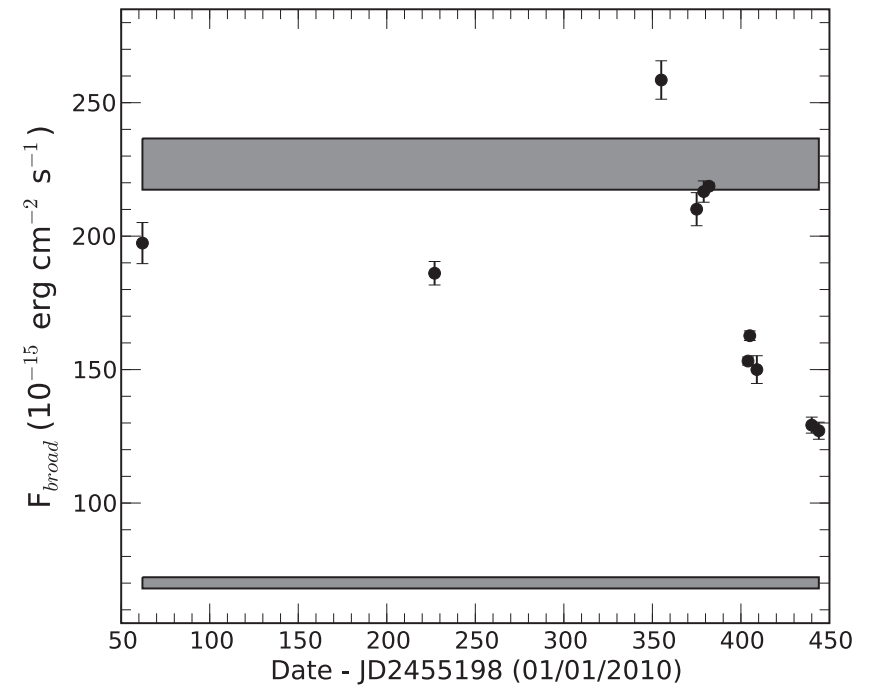

Figure 5. Evolution of the integrated flux of the broad double-peaked $\mathrm{H} \alpha$ line $F_{\text {broad }}$ from 2010 March to 2011 March. The gray horizontal bars show the minimum and maximum values (plus uncertainties) in the spectra from 1991 to 2001 (SB03).

$6000 \mathrm{~km} \mathrm{~s}^{-1}$. A comparison between these peak velocities with those from the period 1991-2001 (SB03) shows that, in the new observations, the blue and red peaks always have larger peak velocities than the minimum values observed between 1991 and 2001 , which are $\approx-2500 \mathrm{~km} \mathrm{~s}^{-1}$ for the blue and $\approx 3250 \mathrm{~km} \mathrm{~s}^{-1}$ for the red, as indicated by the lower gray horizontal bars in both panels of Figure 4. Most velocities are instead similar or higher than the maximum values observed between 1991 and 2001, $\approx-4500 \mathrm{~km} \mathrm{~s}^{-1}$ for the blue and $\approx 4500 \mathrm{~km} \mathrm{~s}^{-1}$ for the red peak. These are indicated by the upper gray horizontal bars in Figure 4 . The values of $V_{B}$ and $V_{R}$ were obtained by using the expression for the relativistic Doppler effect.

Figure 5 shows the variation of the integrated broad $\mathrm{H} \alpha$ emission line flux from 2010 March to 2011 March. The integrated flux is always above the minimum value observed in the period 1991-2003 (lower gray bar in the figure), being close to the maximum value from 2010 March to 2011 January (upper gray bar in the figure). A maximum in the flux occurred between 2010 August 15 and 2010 December 22, when the integrated flux rose above the maximum value observed between 1991 and
2001. After 2010 December 23, there was an abrupt decrease in the broad-line flux, by $\approx 25 \%$ of its maximum value in 20 days. On the other hand, it can be observed that there is little variation on timescales shorter than a week (the maximum time interval between the observations obtained in the same month). Thus, significant variations in the broad-line flux occur on a timescale just above a week. From Figure 5, one can derive a decrease of $\approx 12 \%$ of the flux in 10 days.

Figure 6 shows the temporal evolution of the peak fluxes of the blue and red peaks as well as that of their ratio $F_{B} / F_{R}$. On 2010 March, the red peak was stronger than the blue, while in 2010 August the blue peak was stronger. Thus, within a time interval of 5 months, there has been an inversion of the $F_{B} / F_{R}$ ratio, and, in the following 7 months this ratio decreased to a value that is just below 1, while in 2010 March this value was 0.7. Thus, the change from a stronger red peak to a stronger blue peak was faster than the change back to a stronger red peak. From Figure 6 we thus derive a timescale for the inversion of the relative intensity of the two peaks of $\approx 5$ months.

Figure 7 shows that there is an inverse correlation between the velocity separation of the red and blue peaks $V_{R}-V_{B}$ and $F_{\text {broad }}$, observed both in our recent observations, represented by the circles, as well as in the previous observations of SB03, represented by the crosses. This inverse correlation between $F_{\text {broad }}$ and $V_{R}-V_{B}$ can be interpreted as due to: (1) when the central ionizing source becomes brighter, the line emission from the outer radii become relatively more important, as the radiation of the ionizing source reaches larger distances in the disk, where the velocity is lower; (2) when the central source is fainter, the inner radii are favored, as the ionizing radiation reaches smaller radii, where the velocity is larger.

A comparison of the two data sets in Figure 7 shows that our recent data define a sequence parallel to that of the previous data, displaced from the previous data due to larger velocity separations between the two peaks and to higher fluxes. The larger velocity separation of the two peaks suggests that the line emitting portion of the disk is closer to the ionizing source or has a larger contribution from gas closer to the source.

\section{ACCRETION DISK MODELS}

In order to investigate the origin of the observed variations in the double-peaked profile, we have used accretion disk models 


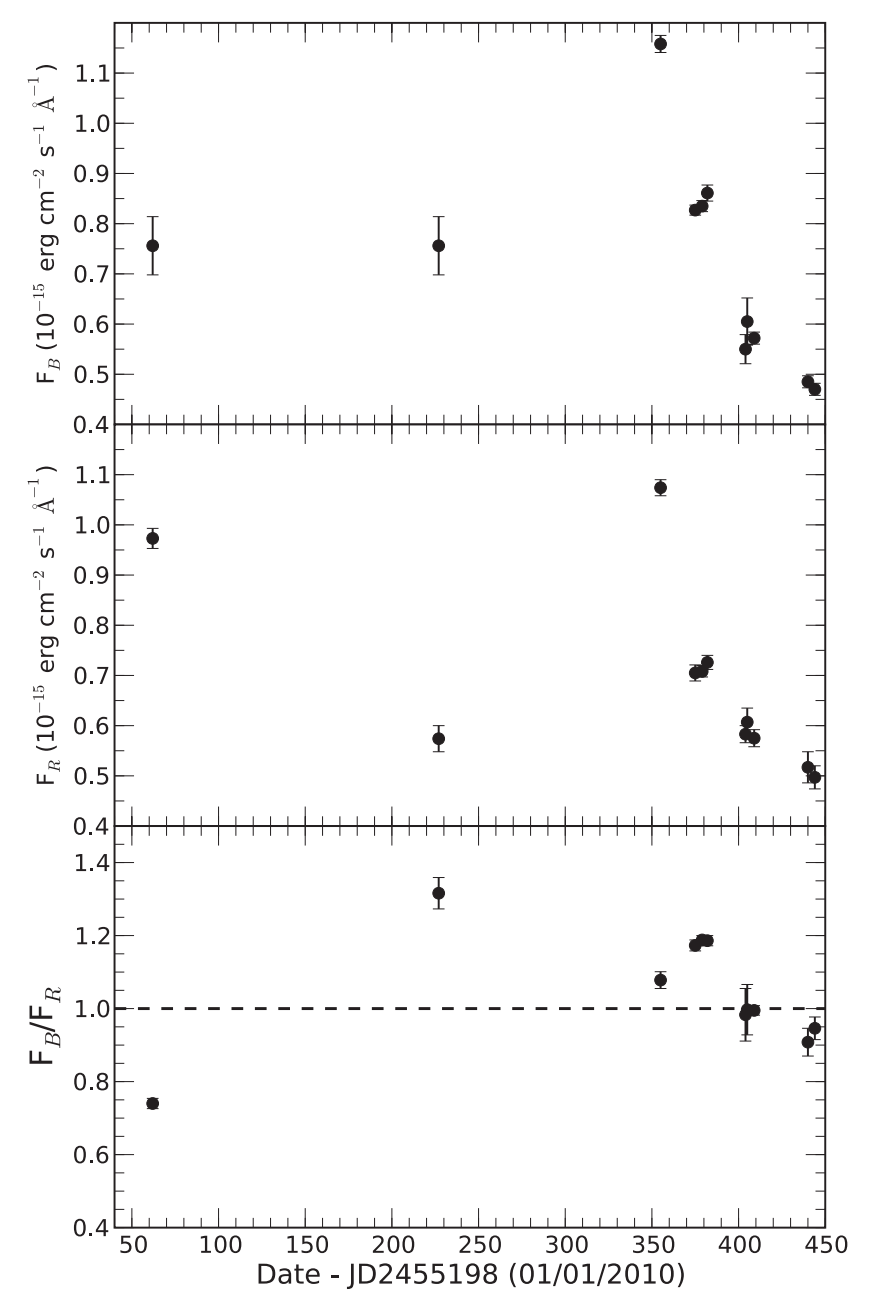

Figure 6. Evolution of the peak flux densities of the blue peak (top panel), the red peak (middle panel), and of the ratio between the two (bottom panel) from 2010 March to 2011 March.

to reproduce the profile. Figure 6 shows that the double-peaked profile still shows variations in the relative intensities of the two peaks, alternating between a stronger blue peak and a stronger red peak, as previously observed by SB03. This pattern of variation supports the origin of the profile in an accretion disk whose emission is not axisymmetric. In SB03, the relative strengths of the two peaks and their variation were better explained by emission from a circular accretion disk with a single spiral arm. The enhanced emission in the arm, as it rotates in the disk, produces the observed asymmetry of the profile. We will thus also adopt in our present modeling the single spiral arm model to fit the double-peaked profiles.

The source of radiation which ionizes the disk in order to drive the $\mathrm{H} \alpha$ emission is also relevant to the modeling. As pointed out in the Introduction, in the case of NGC 1097, this source is an RIAF internal to the line-emitting part of the disk (Nemmen et al. 2006). Dumont \& Collin-Souffrin (1990) have found that, in the case of an accretion disk illuminated by a non-thermal continuum source with a spectral energy distribution extending up to the hard X-ray band, there is a "radius of maximum emission" or "saturation radius," at which the $\mathrm{H} \alpha$ emission reaches a maximum value. Thus, we consider an additional model in which the disk emissivity may increase with radius up to the saturation radius and then decrease outward.

With the above considerations in mind, we then fitted the double-peaked $\mathrm{H} \alpha$ line profiles with two different models,

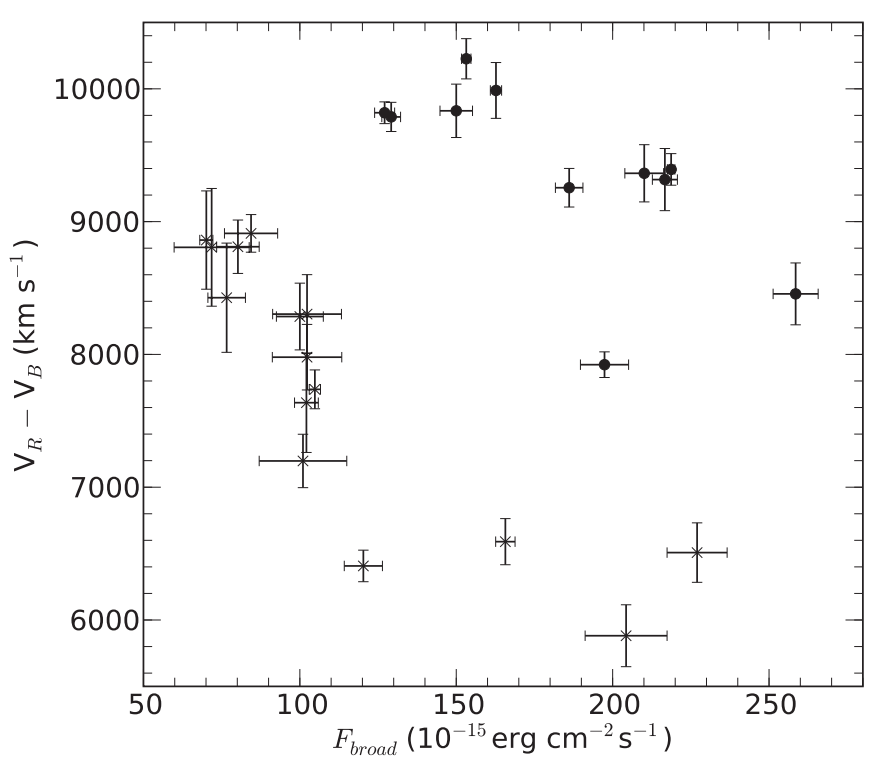

Figure 7. Inverse correlation between $F_{\text {broad }}$ and $V_{R}-V_{B}$. Circles represent the measures for this work and crosses represent the data from SB03. The inverse correlation for the new observations, however, is displaced to larger velocities and fluxes relative to previous observations (SB03). This suggests that emission from the inner parts of the disk became more important relative to that of the outer parts.

described below. In both models we assume a circular emitting disk, or wide ring (as it has a hole in the middle), with a perturbation in the emissivity in the form a spiral arm. In the first model, which we call Spiral Arm model, the variations in the relative strengths of the peaks are controlled by the rotation of the spiral arm, while the separation of the peaks is controlled by an emissivity law that is a simple power-law function of the radius. By changing the power-law index we can weight the emissivity of the disk toward inner regions (leading to a broader profile) or the outer regions (leading to a narrower profile). In the second model, which we call the Saturated Spiral model, we have changed the emissivity law to a broken power-law, which can produce a ring of maximum emissivity between the inner and outer radii. In this case, the separation of the peaks is controlled by the radius of this ring of maximum emissivity.

\subsection{Spiral Arm Model}

We adopt the formulation described in Gilbert et al. (1999), in which: (1) the line emitting portion of the disk is circular and located between an inner radius $\xi_{1}$ and an outer radius $\xi_{2}$ (where $\xi$ is the disk radius in units of the gravitational radius $r_{g}=G M_{\bullet} / c^{2}, c$ is the light speed, $G$ is the gravitational constant and $M_{\bullet}$ is the mass of the black hole); (2) the disk has an inclination $i$ relative to the line of sight (zero degrees is face on). Superimposed on the axisymmetric emissivity of the circular disk, there is a perturbation in the form of a spiral arm. We adopt only one spiral arm, as we have already verified that two or more arms do not reproduce well the variations in the profile (SB03).

The total emissivity of the disk is given by

$$
\begin{aligned}
\epsilon(\xi, \phi)= & \epsilon(\xi)\left\{1+\frac{A}{2} \exp \left[-\frac{4 \ln 2}{\delta^{2}}\left(\phi-\psi_{0}\right)^{2}\right]\right. \\
& \left.+\frac{A}{2} \exp \left[-\frac{4 \ln 2}{\delta^{2}}\left(2 \pi-\phi+\psi_{0}\right)^{2}\right]\right\},
\end{aligned}
$$


Table 3

Parameters for the Spiral Arm Model Fits

\begin{tabular}{lcccr}
\hline \hline Date & $A$ & $q$ & $\begin{array}{c}\phi_{0} \\
(\mathrm{deg})\end{array}$ & $\begin{array}{c}\text { Shift } \\
\left(\mathrm{km} \mathrm{s}^{-1}\right)\end{array}$ \\
\hline 2010 Mar 4 & 2.0 & -2.0 & 130 & 0 \\
2010 Aug 15 & 1.0 & 1.0 & 250 & 0 \\
2010 Dec 22 & 0.3 & 1.0 & 330 & 0 \\
2011 Jan 11 & 0.5 & 1.5 & 340 & -460 \\
2011 Jan 15 & 0.5 & 1.5 & 340 & -460 \\
2011 Jan 18 & 0.5 & 1.5 & 340 & -460 \\
2011 Feb 8 & 1.0 & 2.0 & 360 & -460 \\
2011 Feb 10 & 1.0 & 2.0 & 360 & -460 \\
2011 Feb 14 & 1.0 & 2.0 & 360 & -460 \\
2011 Mar 17 & 2.0 & 2.0 & 400 & -460 \\
2011 Mar 21 & 2.0 & 2.0 & 400 & -460 \\
\hline
\end{tabular}

where

$$
\epsilon(\xi)=\epsilon_{0} \xi^{-q}
$$

is the axisymmetric emissivity of the disk, $A$ is the brightness contrast between the spiral arm and the underlying disk, and the expression between square brackets represents the decay of the emissivity of the arm as a function of the azimuthal distance $\phi-\psi_{0}$ from the ridge line to both sides of the arm, assumed to be a Gaussian function with FWHM $\delta$ (azimuthal width).

The relation between the azimuthal angle $\phi_{0}$ and the angular position $\psi_{0}$ of the ridge of emissivity on the spiral arm is given by

$$
\psi_{0}=\phi_{0}+\frac{\ln \left(\xi / \xi_{\mathrm{sp}}\right)}{\tan p}
$$

where $\phi_{0}$ is the azimuthal angle of the spiral pattern, $p$ is the pitch angle and $\xi_{\mathrm{sp}}$ is the innermost radius of the spiral arm.

The specific intensity from each location in the disk, in the frame of the emitting particle is calculated as

$$
I\left(\xi, \phi, v_{e}\right)=\frac{\epsilon(\xi, \phi)}{4 \pi} \frac{e^{-\left(v_{e}-v_{0}\right)^{2} / 2 \sigma^{2}}}{(2 \pi)^{1 / 2} \sigma},
$$

where $v_{e}$ is the emission frequency, $v_{0}(\mathrm{H} \alpha 6564.6 \AA)$ is the rest frequency and $\sigma$ is the local "broadening parameter" (Chen \& Halpern 1989).

In the fits of the individual profiles, we first used the same set of parameters of SB03, namely: the inner and outer radii of the line emitting portion of the disk $\xi_{1}=\xi_{\text {sp }}=450$ and $\xi_{2}=1600$, the inclination angle $i=34^{\circ}$, broadening parameter $\sigma=1200 \mathrm{~km} \mathrm{~s}^{-1}$, pitch angle $p=50^{\circ}$, and $\delta=70^{\circ}$. In order to reproduce the evolution of the profile we varied the parameters $A, q$, and $\phi_{0}$. The variation of $\phi_{0}$ regulates the evolution of the shape of the profile, while a variation in $A$, together with $\phi_{0}$, regulates the relative intensities of the two peaks. The value of $q$ regulates the radius in the disk of the maximum line luminosity, which corresponds to the inner regions for the broadest profiles, or to the outer regions for the narrowest profiles.

The best-fitting models are compared to the observed line profiles in Figure 8, while the corresponding parameter values $\left(A, q\right.$, and $\left.\phi_{0}\right)$ are listed in Table 3 . The contrast of the spiral arm $A$ ranged from 2.0 at the first epoch, decreased to a minimum value of 0.5 in 2011 January, and increased again to 2.0 in 2011 March, while $q$ increased from the first to the last epochs. An abrupt variation in $q$ occurred between 2010 March and 2010 August when the value of $q$ changed from -2.0 , favoring the outer parts of the disk, to 1.0, favoring the inner parts of the disk. The $\phi_{0}$ values were constrained to provide a monotonic

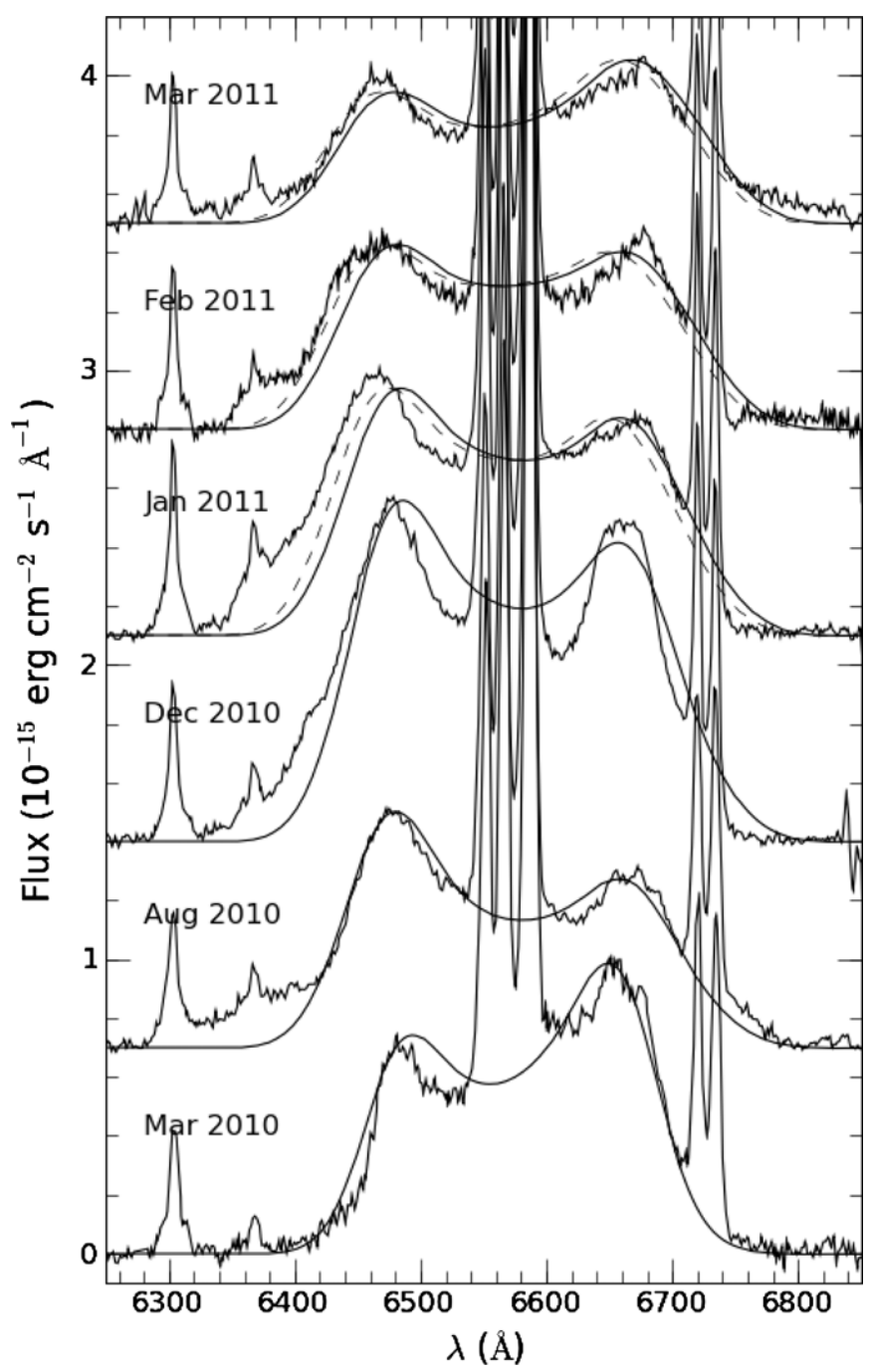

Figure 8. Best fits of the spiral arm model for the epochs from 2010 March 4 to 2011 March 21 , obtained by varying $\phi_{0}$ (orientation of the spiral pattern), $A$ (contrast between the spiral arm and the disk), and $q$ (the slope of the emissivity law). The values of the parameters are listed in Table 3.

rotation of the spiral pattern, from $130^{\circ}$ to $400^{\circ}$. The spiral arm has thus almost completed one revolution in one year.

For the spectra from 2011 January to 2011 March, an improvement of the fit can be obtained by allowing a small, ad hoc blueshift of the central wavelength of the profile relative to the wavelength of the narrow $\mathrm{H} \alpha$ line $(6564.6 \AA)$. This blueshift is $\approx-460 \mathrm{~km} \mathrm{~s}^{-1}$, as listed in the last column of Table 3 . The improved fits with the blueshifts are shown as dashed lines in Figure 8.

In Figure 9 we show the evolution of the $\phi_{0}$ values obtained from the fits, represented by the circles in the figure, while the black solid line is the best linear regression to the points and the gray band covers the uncertainty in the regression. The linear regression has angular coefficient $\dot{\phi}_{0}=0.680 \pm 0.02$ day $^{-1}$, which represents the angular velocity of the arm and implies a rotation period of $P \approx 17.7 \pm 0.5$ months.

\subsection{Saturated Spiral Model}

In an effort to improve the fit to the broad double-peaked $\mathrm{H} \alpha$ profiles, we modified the spiral arm model to allow a more flexible radial emissivity law. We have chosen an emissivity law that allows the existence of a "radius of maximum emissivity" 


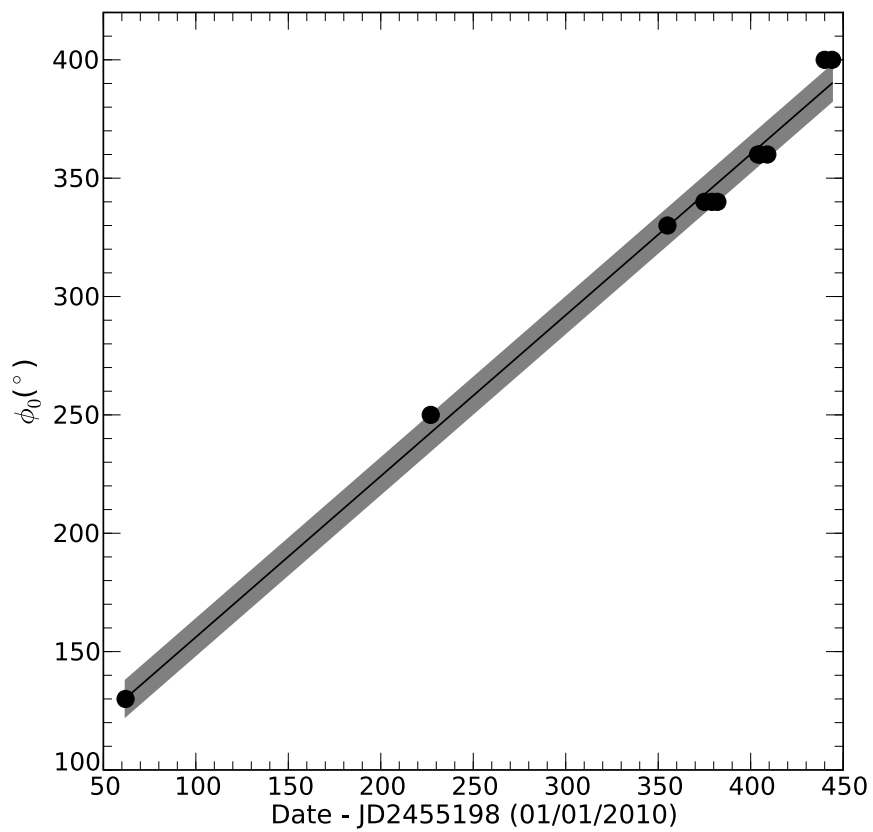

Figure 9. Evolution of the $\phi_{0}$ values obtained from the fit of the spiral arm model. The solid line is a linear fit to the data and the shaded bar covers the uncertainty in the fit.

between the inner and outer radii of the disk at which the broad $\mathrm{H} \alpha$ line reaches a maximum intensity, as proposed by Dumont $\&$ Collin-Souffrin (1990). The emissivity increases with radius until the saturation radius and then decreases outward.

The radius of maximum emissivity was incorporated by changing the axisymmetric part of the emissivity of the disk (Equation (2)) as follows:

$$
\epsilon(\xi)=\left\{\begin{array}{ll}
\epsilon_{0} \xi^{-q_{1}}, & \xi_{1}<\xi<\xi_{q} \\
\epsilon_{0} \xi_{q}^{-\left(q_{1}-q_{2}\right)} \xi^{-q_{2}}, & \xi_{q}<\xi<\xi_{2}
\end{array},\right.
$$

where the new parameter $\xi_{q}$ is the radius of maximum emissivity, or saturation radius, at which the emissivity law changes; $q_{1}$ is the index of the emissivity law for the regions internal to $\xi_{q}$ $\left(\xi_{1}<\xi<\xi_{q}\right) ; q_{2}$ is the index for the regions external to $\xi_{q}\left(\xi_{q}<\xi<\xi_{2}\right) ; \xi_{1}$ and $\xi_{2}$ are the inner and outer radii, respectively.

We kept the value $q_{2}=3.0$, as discussed by Dumont \& Collin-Souffrin (1990), who showed that beyond the saturation radius the flux emitted by the line decreases as $\propto \xi^{-3}$. After many tests, $q_{1}=-2.0$ gave the best fits, and was fixed at this value. The negative value allows the increase of the emissivity until the saturation radius. The parameter $\xi_{q}$ was allowed to vary. We kept the parameters of the spiral arm at the values $\xi_{\text {sp }}=\xi_{1}, p=50^{\circ}, \delta=70^{\circ}$, as well as the inclination of the disk at $i=34^{\circ}$.

We have varied the broadening parameter and found that the best value to reproduce the shape of most profiles was $\sigma=900 \mathrm{~km} \mathrm{~s}^{-1}$.

In summary, in the Saturated Spiral model, the only parameters we needed to vary in order to reproduce the evolution of the profile were $\xi_{q}, A$, and $\phi_{0}$. The changes in the separation between the blue and red peaks were obtained by changing $\xi_{q}$; when $\xi_{q}$ is smaller, the separation between the blue and red peaks increases.

The best-fitting models are compared with the observed line profiles in Figure 10, and the corresponding parameters values $\left(\xi_{q}, A\right.$, and $\left.\phi_{0}\right)$ are listed in Table 4 . The parameter $\xi_{q}$ ranged

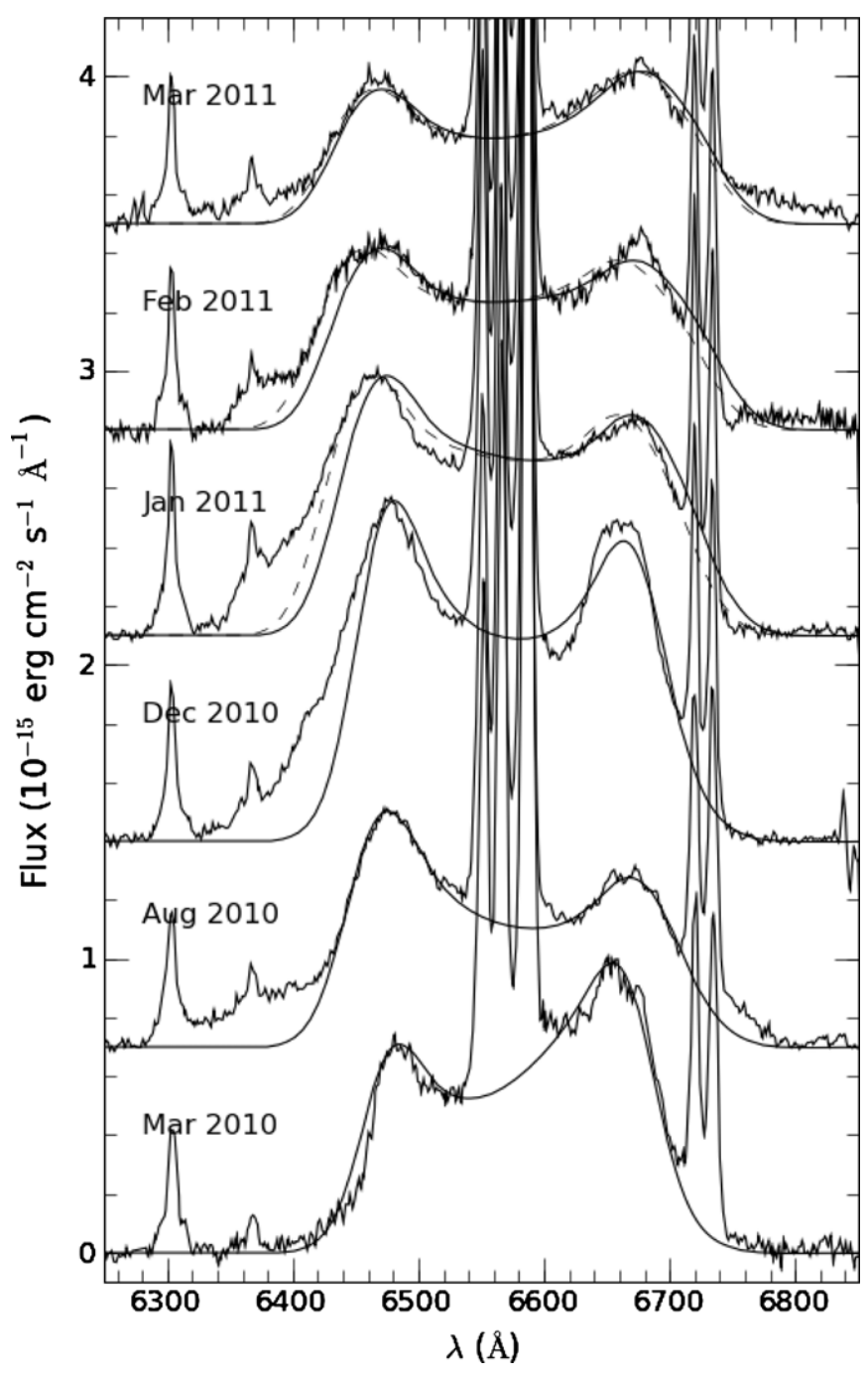

Figure 10. Best fits for the hybrid model, obtained by varying $\phi_{0}$ (orientation of the spiral pattern), $A$ (contrast between the disk and the spiral arm), and $\xi_{q}$ (radius of maximum emissivity). The values of the parameters are listed in Table 4.

Table 4

Parameters for the Saturated Spiral Model Fits

\begin{tabular}{lcrcr}
\hline \hline Date & $A$ & $\xi_{q}$ & $\begin{array}{c}\phi_{0} \\
(\mathrm{deg})\end{array}$ & $\begin{array}{c}\text { Shift } \\
\left(\mathrm{km} \mathrm{s}^{-1}\right)\end{array}$ \\
\hline 2010 Mar 4 & 3.0 & 1200 & 140 & 0 \\
2010 Aug 15 & 1.0 & 800 & 220 & 0 \\
2010 Dec 22 & 0.2 & 1000 & 330 & 0 \\
2011 Jan 11 & 1.0 & 650 & 340 & -500 \\
2011 Jan 15 & 1.0 & 650 & 340 & -500 \\
2011 Jan 18 & 1.0 & 650 & 340 & -500 \\
2011 Feb 8 & 1.0 & 550 & 360 & -550 \\
2011 Feb 10 & 1.0 & 550 & 360 & -550 \\
2011 Feb 14 & 1.0 & 550 & 360 & -550 \\
2011 Mar 17 & 1.5 & 600 & 400 & -230 \\
2011 Mar 21 & 1.5 & 600 & 400 & -230 \\
\hline
\end{tabular}

from a maximum value of 1200 in 2010 March when the separation between the peaks was the smallest, to a minimum value of 550-600 in 2011 February-March, when the separation between the peaks was the largest. The parameter $A$ assumed the value 3.0 in 2010 March, when the red peak was significantly stronger than the blue, as seen in Figure 10, while in 2010 
December this parameter reached the minimum value of 0.2 , when the peaks had similar strengths. Similar to the previous model, $\phi_{0}$ varied from $140^{\circ}$ in the first epoch to $400^{\circ}$ in the last.

As in the case of the Spiral Arm model, from 2011 January to 2011 March, the fit was improved by introducing a blueshift which is listed in the last column of Table 4. This blueshift ranges from $\approx-230$ to $-550 \mathrm{~km} \mathrm{~s}^{-1}$, and the improved fits with the blueshifts are shown as dashed lines in Figure 10.

In Figure 11 we show emissivity maps constructed using the parameters of the best fits using the Saturated Spiral model. In 2010 March the radius of maximum emissivity $\xi_{q}$ was closer to $\xi_{2}$, when the outer parts where brighter, while in 2011 March $\xi_{q}$ was closer to $\xi_{1}$ and the inner parts were brighter.

\section{DISCUSSION}

\subsection{Timescales}

In order to discuss the physical processes driving the changes in the profiles, we first review four relevant timescales for the accretion disk in NGC 1097: the viscous timescale $\left(\tau_{\mathrm{visc}}\right)$, the sound-crossing timescale $\left(\tau_{s}\right)$, the dynamical timescale $\left(\tau_{\mathrm{dyn}}\right)$, and the light travel timescale $\left(\tau_{l}\right)$. These timescale are presented in Frank et al. (2002) and can be modified in order to express the disk radius in terms of the gravitational radius (Eracleous 1998; Lewis et al. 2010):

$$
\begin{gathered}
\tau_{\text {visc }}=10^{6} M_{8}^{3 / 2} \xi_{3}^{5 / 4} \alpha_{-1}^{-4 / 5} \dot{M}_{-1}^{-3 / 10} \text { years } \\
\tau_{s}=70 M_{8} \xi_{3} T_{5}^{-1 / 2} \text { years } \\
\tau_{\text {dyn }}=6 M_{8} \xi_{3}{ }^{3 / 2} \text { months } \\
\tau_{l}=6 M_{8} \xi_{3} \text { days }
\end{gathered}
$$

where $\xi_{3}=10^{-3} \xi, M_{8}=M_{\bullet} \times 10^{-8} M_{\odot}^{-1}, \alpha=10^{-1} \times \alpha_{-1}$ is the Shakura-Sunyaev viscosity parameter, $T_{5}$ is the temperature in units of $10^{5} \mathrm{~K}$, and $\dot{M}_{-1}$ is the mass accretion rate in units of $0.1 M_{\odot} \mathrm{yr}^{-1}$.

For NGC $1097, M_{\bullet}=1.2 \times 10^{8} M_{\odot}$ (Lewis \& Eracleous 2006), and $\dot{M} / \dot{M}_{\text {Edd }}=0.0064$ (in Eddington units; Nemmen et al. 2006), corresponding to $\dot{M}=0.018 M_{\odot} \mathrm{yr}^{-1}$. Adopting typical values of $\alpha=0.1$ and $T_{5}=0.1$, a characteristic radius for the disk is $\xi=1000$. Adopting the above values of $M_{\bullet}$ and $\dot{M}$, we obtain the following estimates for the timescales:

1. $\tau_{\text {visc }} \gtrsim 10^{7}$ years: this large value for the viscous timescale does not support that the observed changes in the profile - on timescales from weeks to years - are due to inward transfer of mass due to viscous torques;

2. $\tau_{s} \sim 265$ years: this is also much larger than the observed variations timescales;

3. $\tau_{\text {dyn }} \gtrsim 7$ months: the dynamical timescale depends on the radius, with the inner parts of the disk having shorter timescales than the outer parts. Adopting $\xi_{3}=0.45$ for the inner radius, as obtained from our modeling, the corresponding dynamical timescale is just above 2 months. The new observations presented here show that the variation timescale of the relative strengths of the blue and red peaks $\left(F_{B} / F_{R}\right)$ is $\approx 5$ months (see Figure 6 ), thus compatible with the dynamical time. In our modeling, this variation was reproduced by the rotation of the spiral pattern around the disk, whose period is close to the dynamical time at $\xi_{2}$;
4. $\tau_{l} \gtrsim 7$ days: considering the range of radii of the disk, this timescale ranges from $\approx 4$ to $\approx 12$ days. This is the shortest timescale of the disk. According to our data (see Figure 5), the smallest timescale of the profile variations-namely variations in the integrated flux of the double-peaked line-occur in $\leqslant 7$ days. This supports the interpretation that the variability in the integrated flux $F_{\text {broad }}$ occurs in the light travel timescale, as a result of variations in the ionizing flux. Similarly, the variations in $V_{B}$ and $V_{R}$ (see Figure 4) that correlate with $F_{\text {broad }}$ also occur in the light travel timescale. According to the models, these are due to changes in the region of maximum emissivity of the disk.

\subsection{Constraints on the Physical Mechanisms Causing the Variations}

The timescales discussed above provide constraints on the mechanisms that cause the changes in the double-peaked profile. Our data clearly reveal two distinct timescales: (1) the first corresponding to changes in the integrated flux of the doublepeaked profile, which is just over 7 days; and (2) the second corresponding to the changes in relative strengths of the blue and red peaks, which is in the range 5-6 months.

We have identified the first timescale with the light travel timescale. In order to model the corresponding variations in the flux of the double-peaked line, and at the same time reproduce the variation of the width of the profiles it was necessary to vary the disk emissivity. When the flux is higher, the profile is narrower, and the emissivity is higher in the outer parts of the disk, while when the flux is lower, the profile is broader and the emissivity is relatively higher in the inner parts of the disk. In the Saturated Spiral model, what varies is the radius of maximum emissivity.

The physical scenario we favor is the following: the doublepeaked profile originates in gas rotating in a thin accretion disk, which is ionized by an RIAF (Nemmen et al. 2006) located inside the inner radius of the line-emitting portion of the disk. As the variations occur on the light travel timescale, our interpretation is that the variation in $F_{\text {broad }}$ can be considered a "reverberation" effect produced by variations in the ionizing flux from the central RIAF, and allows a test for the values of the radii obtained via the models. Calculating the values for the inner and outer radii of the disk in light days, we obtain for $\xi_{1}$ $\approx 3$ light days and for $\xi_{2} \approx 11$ light days, which is consistent with the variability timescale of $\approx 7$ days that we observe.

In order to further confirm the reverberation hypothesis, it would be necessary to do a proper "reverberation mapping campaign" (e.g., Peterson et al. 2004) of the accretion disk via simultaneous observations of the RIAF emission (e.g., in $\mathrm{X}$-rays) and the double-peaked profile. We plan to pursue such a monitoring campaign in the future, including a more extended spectral coverage to include the blue part of the optical spectrum, which will also allow us to test other models for the origin of the continuum, such as the one proposed by Gaskell (2011).

We have identified the second timescale-of the variations in the relative strengths of the blue and red peaks-with the dynamical timescale. This means that the asymmetry in the profile is due to a non-axisymmetric emitting structure-modeled as a single spiral arm-rotating in the disk, with a rotation period of $\approx 18$ months. This timescale and its interpretation differs from our previous one (SB03). In that paper we concluded that the variation of the relative strengths of the two peaks occurred in the sound-crossing timescale. This distinct interpretation was 
SATURATED SPIRAL MODEL
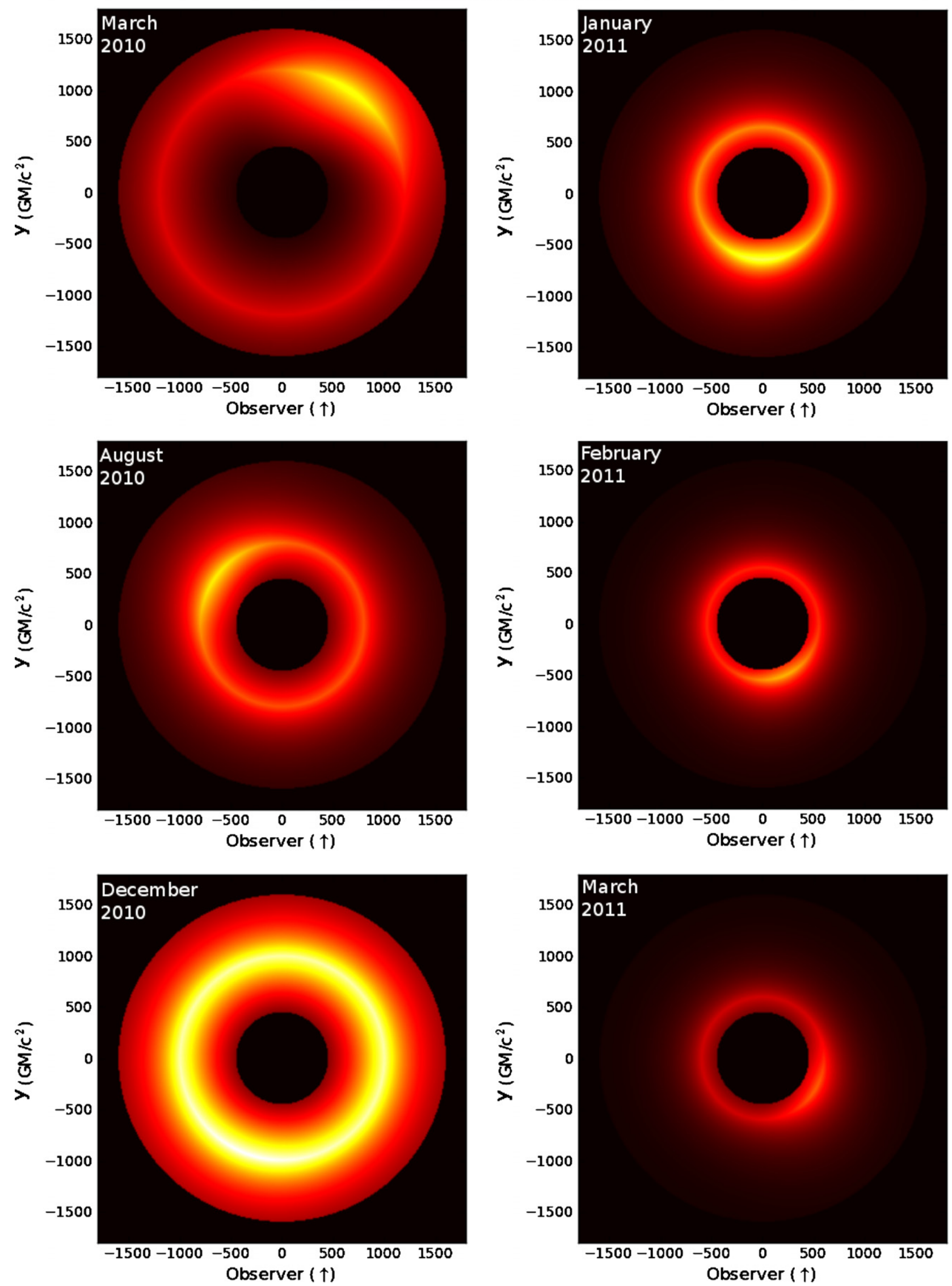

Figure 11. Images showing the disk emissivity from 2010 March to 2011 March, using the hybrid model. White represents the brightest regions, and the observer is to the bottom. The disk parameters for these epochs are listed in Table 4.

(A color version of this figure is available in the online journal.) 
due to the fact that the period we had derived then for the variation was $5.5 \mathrm{yr}$, and at that time our estimate for the SMBH mass was $\approx 10^{6} M_{\odot}$, giving a smaller value for the sound-crossing timescale, consistent with the derived period of variation. With a revised SMBH mass two orders of magnitude larger, the revised sound-crossing timescale is much larger than the timescale of the variation of the relative fluxes of the blue and red peaks we have now derived from our more frequent observations.

Is important to point out that the perturbation does not need to be a spiral arm, even though we used the spiral arm prescription to model it. In the images of the disk, constructed using the best model parameters for the different epochs, shown in Figure 11, the spiral arm actually looks more like an azimuthal arc. Therefore, it could be that this arc is actually a clump that was produce by self-gravity and then sheared (see discussion in Flohic \& Eracleous 2008). Another alternative is that this feature is an irradiation-induced warp (Pringle 1996; Maloney et al. 1996). But although the irradiation-induced warp model can reproduce the observed variability of the line profiles (Wu et al. 2008), the required rotation timescale is incompatible with the mass of the SMBH in NGC 1097 (see Equation (4) in Storchi-Bergmann et al. 1997). On the other hand, the sheared clump would orbit the SMBH on the dynamical timescale and therefore could reproduce the 5 month variation that we observe.

Figure 7 shows that the profiles got broader and brighter in the recent (2010-2011) observations when compared with the previous ones (1991-2001), although at both epochs there is an inverse correlation between the flux and width of the profiles. This inverse correlation is expected in the reverberation scenario discussed above, but there is an offset between the previous and present correlations. A comparison between the parameters of the present and previous spiral arm model shows that the emissivity law in the recent epochs favors a higher contribution of the inner regions relative to the outer regions of the disk, leading to a broader profile. One possibility to explain this increase in emissivity of the innermost regions is an increase in the gas density in these regions of the disk in the most recent epochs when compared with the period 1991-2001.

A comparison between Figures 8 and 10 shows that the Saturated Spiral model gives a better fit to the double-peaked profiles. In order to quantify the goodness of the fits, we have obtained the root mean square (rms) deviation between the observed profiles and the models. In almost all observations the Saturated Spiral model provided a smaller rms when compared with that for the Single Spiral arm model, confirming what is seen in the fits.

Finally, we point out that, although the models can in general reproduce the $\mathrm{H} \alpha$ profiles, there are several epochs in which we observe an excess flux in the blue wings of the profiles relative to the models. This excess flux is observed from 2010 August to 2011 February (Figures 8 and 10). In addition, from 2011 January to March we observe a systematic blueshift of the center of the line, from -230 to $-550 \mathrm{~km} \mathrm{~s}^{-1}$ which could be due to an accretion disk wind. Therefore, it will be worthwhile in future work to compare the observed line profiles with models that include an outflowing wind (e.g., Hall \& Chajet 2010).

\subsection{The Mass of the Supermassive Black Hole}

The physical value of the disk timescales, such as the lighttravel timescale $\left(\tau_{l}\right)$, as well as the inner and outer radii of the accretion disk, are strongly dependent on the mass of the $\mathrm{SMBH}$. Thus, uncertainties in its determination must be taken into account. We have adopted here the SMBH mass value of Lewis \& Eracleous (2006): $M_{\bullet}=1.2 \pm 0.2 \times 10^{8} M_{\odot}$.

The range of values for the inner and outer radii of the accretion disk ( $\xi_{1}=450$ and $\xi_{2}=1600$, respectively) resulting from the uncertainties in the SMBH mass quoted by Lewis $\&$ Eracleous (2006) are $2.7 \leqslant \xi_{1} \leqslant 3.6$ light-days and $9.1 \leqslant \xi_{2} \leqslant 12.8$ light-days. But Lewis \& Eracleous (2006) obtain the value for the SMBH mass using the $M_{\bullet}-\sigma$ relation of Tremaine et al. (2002), pointing out that they do not take into account the scatter in this relation. They consider only the uncertainty in the coefficients of the relation that results from the scatter (effectively they assume that the observed scatter is not intrinsic). If we now consider that the uncertainty in the SMBH mass is also affected by the scatter in the $M_{\bullet}-\sigma$ relation from Tremaine et al. (2002), which can introduce an uncertainty in the mass of $\approx 0.3 \mathrm{dex}$, the resulting minimum and maximum values for the inner and outer radii become: (1) for $M_{\bullet} \approx 0.6 \times 10^{8} M_{\odot}$, the minimum radii are $\xi_{1} \approx 1.5$ light-days and $\xi_{2} \approx 5.5$ lightdays; and (2) for $M_{\bullet} \approx 2.4 \times 10^{8} M_{\odot}$, the maximum radii are $\xi_{1} \approx 6$ light-days and $\xi_{2} \approx 22$ light-days.

Thus, even considering the maximum uncertainties which could affect the determination of the SMBH mass, the resulting values for the inner and outer radii of the disk are still of the order of light-days. The above results support the interpretation that the shortest variation timescale of 7 days is the light-travel time between the ionizing source at the center of the disk and the mean radius of the disk. In fact, this observed timescale can actually constrain the values of $\xi_{1}$ to $\leqslant 7$ light-days and of $\xi_{2}$ to $\geqslant 7$ light-days.

In addition, if we assume that 7 light-days is the mean distance between the broad-line-emitting clouds and the SMBH, we can estimate the mass of the SMBH independently from the model, as follows. If this system of clouds is flattened, as suggested by their double-peaked profile, and we adopt as the corresponding Keplerian velocity around the SMBH the mean velocity of the peaks_ $\approx 4650 \mathrm{~km} \mathrm{~s}^{-1}$ —we obtain $M_{\bullet}=1.1 \times 10^{8} M_{\odot}$, for an inclination of $34^{\circ}$ relative to the plane of the sky (as obtained from the disk model). This value is in good agreement with the one obtained by Lewis \& Eracleous (2006).

\section{CONCLUSIONS}

We report the discovery of short timescale variations in the broad double-peaked $\mathrm{H} \alpha$ emission line from the LINER nucleus of NGC 1097, observed in 9 epochs between 2010 March and 2011 March. They comprise: (1) variations in the integrated flux and width of the line over a timescale of $\geqslant 7$ days, which we identified as the light-travel time between the ionizing source and the disk; it is the first time that such short timescale variations have been seen in a double-peaked profile from the nucleus of a galaxy; and (2) variations in the relative intensity of the blue and red peaks on a timescale of 5-6 months, which is compatible with the disk dynamical timescale.

Using two distinct accretion disk models, we reproduced these variations through the combination of two effects:

1. short timescale changes in the disk emissivity-namely in the contrast $A$ and index $q$ in the Spiral Arm model and $A$ and $\xi_{q}$ in the Saturated Spiral model;

2. the rotation of the spiral arm-via the change of the orientation $\phi_{0}$ of the arm in both models-resulting in a estimate for the rotation period of the arm of $\approx 18$ months.

The values of the inner and outer radii obtained from previous modeling were kept in the present modeling and are, 
respectively, $\xi_{1}=450$ and $\xi_{2}=1600$ (corresponding to $\approx 3$ and 11 light-days, respectively). These values are in good agreement with the short timescale variations seen in our observations. This agreement supports the identification of the shortest timescale variations with the light travel time between the ionizing source and the accretion disk, in one hand, and gives further support to the model and the derived parameters, on the other hand.

Our recent observations support the scenario in which an accretion disk whose emissivity changes due to reverberation of a variable ionizing continuum (presumably from an RIAF) has a non-axisymmetric feature rotating in the dynamical timescale.

We thank the referee, Martin Gaskell, for the careful reading of the manuscript and his thoughtful comments. Based on observations obtained at the Gemini Observatory, which is operated by the Association of Universities for Research in Astronomy, Inc., under a cooperative agreement with the NSF on behalf of the Gemini partnership: the National Science Foundation (United States), the Science and Technology Facilities Council (United Kingdom), the National Research Council (Canada), CONICYT (Chile), the Australian Research Council (Australia), Ministrio da Ciência, Tecnologia e Inovação (Brazil), and Ministerio de Ciencia, Tecnología e Innovación Productiva (Argentina). J.S.S. and T.S.B. acknowledge the Brazilian institutions CNPq, CAPES, and FAPERGS for partial support.

\section{REFERENCES}

Bica, E. 1988, A\&A, 195, 76

Bower, G. A., Wilson, A. S., Heckman, T. M., \& Richstone, D. O. 1996, AJ, 111,1901

Chen, K., \& Halpern, J. P. 1989, ApJ, 344, 115

Dumont, A. M., \& Collin-Souffrin, S. 1990, A\&A, 229, 313

Eracleous, M. 1998, Adv. Space Res., 21, 33
Eracleous, M., \& Halpern, J. P. 1994, ApJS, 90, 1

Eracleous, M., Livio, M., Halpern, J. P., \& Storchi-Bergmann, T. 1995, ApJ, 438,610

Flohic, H. M. L. G., \& Eracleous, M. 2008, ApJ, 686, 138

Frank, J., King, A., \& Raine, D. J. 2002, in Accretion Power in Astrophysics (3rd ed.; Cambridge: Cambridge Univ. Press)

Gaskell, C. M. 2010, arXiv:1008.1057

Gaskell, C. M. 2011, Balt. Astron., 20, 392

Gaskell, C. M., Klimek, E. S., \& Nazarova, L. S. 2007, BAAS, 39, 947

Gezari, S., Halpern, J. P., \& Eracleous, M. 2007, ApJS, 169, 167

Gilbert, A. M., Eracleous, M., Filippenko, A. V., \& Halpern, J. P. 1999, in ASP Conf. Ser. 175, Structure and Kinematics of Quasar Broad Line Regions, ed. C. M. Gaskell, W. N. Brandt, M. Dietrich, D. Dultzin-Hacyan, \& M. Eracleous (San Francisco, CA: ASP), 189

Hall, P. B., \& Chajet, L. S. 2010, in IAU Symp. 267, Co-Evolution of Central Black Holes and Galaxies, ed. B. M. Peterson, R. S. Somerville, \& T. StorchiBergmann (Cambridge: Cambridge Univ. Press), 398

Heckman, T. M. 1980, Highlights Astron., 5, 185

Ho, L. C., Rudnick, G., Rix, H.-W., et al. 2000, ApJ, 541, 120

Lewis, K. T., \& Eracleous, M. 2006, ApJ, 642, 711

Lewis, K. T., Eracleous, M., \& Storchi-Bergmann, T. 2010, ApJS, 187, 416

Maloney, P. R., Begelman, M. C., \& Pringle, J. E. 1996, ApJ, 472, 582

Narayan, R., \& McClintock, J. E. 2008, New Astron. Rev., 51, 733

Nemmen, R. S., Storchi-Bergmann, T., Yuan, F., et al. 2006, ApJ, 643, 652

Peterson, B. M., Ferrarese, L., Gilbert, K. M., et al. 2004, ApJ, 613, 682

Pringle, J. E. 1996, MNRAS, 281, 357

Shields, J. C., Rix, H.-W., McIntosh, D. H., et al. 2000, ApJ, 534, L27

Storchi-Bergmann, T., Baldwin, J. A., \& Wilson, A. S. 1993, ApJ, 410, L11

Storchi-Bergmann, T., Eracleous, M., Livio, M., et al. 1995, ApJ, 443, 617

Storchi-Bergmann, T., Eracleous, M., Ruiz, M. T., et al. 1997, ApJ, 489, 87

Storchi-Bergmann, T., Nemmen da Silva, R., Eracleous, M., et al. 2003, ApJ, 598,956

Strateva, I. V., Brandt, W. N., Eracleous, M., \& Garmire, G. 2008, ApJ, 687, 869

Strateva, I. V., Brandt, W. N., Eracleous, M., Schneider, D. P., \& Chartas, G. 2006, ApJ, 651, 749

Tremaine, S., Gebhardt, K., Bender, R., et al. 2002, ApJ, 574, 740

Veilleux, S., \& Zheng, W. 1991, ApJ, 377, 89

Wu, S.-M., Wang, T.-G., \& Dong, X.-B. 2008, MNRAS, 389, 213

Yee, H. K. C., \& Oke, J. B. 1981, ApJ, 248, 472

Zheng, W., Veilleux, S., \& Grandi, S. A. 1991, ApJ, 381, 418 